\title{
Longitudinal Beam Response Measurements at CESR ${ }^{*}$
}

\author{
John M. Byrd \\ Laboratory of Nuclear Studies, Cornell University \\ Ithaca, NY 14853 USA
}

\begin{abstract}
Measurements of the the beam transfer function (BTF) for longitudinal dipole motion of multiple electron bunches are presented. Quantitative agreement is found for low current measurements at various bunch lengths. Qualitative comparisons are made between the behavior of different multibunch modes at low and high current.
\end{abstract}

\section{Introduction}

Longitudinal beam stability historically has not been a problem at CESR but with the prospcet of future upgrades and operation at higher current levels, we have begun a series of experiments to measure the effect of the longitudinal impedance on the longitudinal dipole motion of a single beam of single and multiple bunches of $e \pm$ and estimate the multibunch stability limit. Beam transfer function methods have been used successfully in the past to measure the impedance for coasting beams $[1,2]$ and have been measured for bunched beams [3].

We excited the beam by modulating the RF phase and sweeping the excitation frequency through the synchrotron sidcbands of revolution harmonics to drive particular longitudinal multibunch modes. The excitation and beam response were recorded by a LeCroy Transient digitizer operating as a network analyzer[4] and used to compute the longitudinal BTF, yielding both amplitude and phase response of the beam. The data acquisition system also allowed for measurement of motion of individual bunches in multibunch operation. Longitudinal beam motion was detected as transverse displacement due to coherent energy oscillations at a point of finite dispersion in the lattice. $\mathrm{BTF}$ measurements were made for all dipole multibunch modes for 3 and 7 bunches vs. current and vs. bunch length. Measurements of the transient response at low currents to impulse excitations were also used to measure Landau damping due to the spread in synchrotron frequencies resulting from the nonlinearity of the RF restoring force.

*Work supported by the National Science Foundation

\section{Theory}

\section{Multibunch Mode Spectrum}

For a beam of $\mathrm{M}$ equally spaced and equally populated bunches, the multibunch mode frequencies are given by

$$
f_{\ell}=(n+\ell M) f_{0}+m f_{s 0}
$$

where $n=-\infty, \ldots, \infty, f_{0}$ is the revolution frequency, $f_{s 0}$ is the synchrotron frequency, $\ell$ is the multibunch mode number and $m$ specifies the mode of oscillation within the bunch. Each multibunch mode is characterized by an instantaneous bunch-bunch phase difference of $\Delta \phi=\frac{2 \pi}{\mathrm{M}} \ell$. This paper will be limited to discussion of the dipole $(\mathrm{m}=1)$ mode.

\section{Multibunch Beam Transfer Function $[5,6]$}

In the presence of an external driving term the longitudinal equation of motion of a single particle in a bunch, neglecting radiation damping, can be expressed as

$$
\ddot{\tau}+\omega_{s 0}^{2} \tau=g_{0} e^{j \omega t}
$$

where $\tau$ is the time deviation of the particle from the synchronous particle, $\omega_{s 0}^{2}=\frac{\omega_{0}^{2} h \alpha e V_{r, p} \cos \phi_{s}}{2 \pi E}, \alpha$ is the momentum compaction, $\frac{\omega_{0}}{2 \pi}$ is the revolution frequency, $\mathrm{E}$ is the particle energy, $\phi_{s}$ is the synchronous phase angle, $V_{r f}$ is the $\mathrm{RF}$ voltage, and $\mathrm{h}=\frac{\omega_{\mathrm{rI}}}{\omega_{0}}$ is the harmonic number. $g_{0}$ and $\omega$ are the amplitude and frequency of the driving term due to the phase modulation.

For multiple bunches, the net coherent motion of a mode can be found using a linearized Vlasov equation approach. The coherent motion of mode $\ell$ is given as $\bar{\gamma}_{\ell}(t)=\left|\bar{\gamma}_{\ell}\right| c^{j \omega t}$ and the measured beam response is $\dot{\bar{\tau}}_{\ell}(t)=j \omega \bar{\tau}_{\ell}(t)$. The $\mathrm{BTF}$ is given as the ratio of the beam response to the external driving term in the frequency domain.

$$
\frac{\left|\dot{\bar{\tau}}_{\ell}\right|}{g_{0}}=I
$$

where I is the dispersion integral

$$
I=\frac{\pi}{2} \int_{0}^{\infty} d a \frac{\frac{\partial \psi_{0}}{\partial a}}{\omega-\omega_{s}(a)}
$$




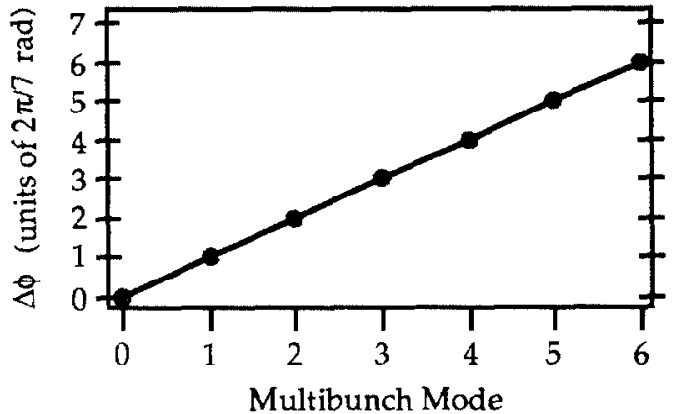

Figure 1: Measured Bunch-bunch phase difference vs. mode number. Error bars are too small to show.

a is the amplitude of longitudinal oscillations such that $\tau=$ $a \cos \omega_{s} t$ and $\dot{\tau}=-\omega_{s} a \sin \omega_{s} t$, and $\psi_{0}(a)=\frac{1}{2 \pi \sigma_{r}^{2}} \exp \left(\frac{-a^{2}}{2 \sigma_{\tau}^{2}}\right)$ is the equilibrium phase space density.

The nonlinearity of the RF bucket is assumed to yield an amplitude-dependent synchrotron frequency of the form

$$
\omega_{s}(a)=\omega_{s 0}-\kappa\left(\frac{a}{\sigma_{\tau}}\right)^{2}
$$

where $c \sigma_{\tau}=\frac{c \alpha}{\omega_{0}} \sigma_{\epsilon}$ is the RMS bunch length, $\sigma_{\epsilon}$ is the relative bunch energy spread, and $\kappa$ is a weak function of $\phi_{s}$.

The beam-induced voltage per turn due to the coherent motion is

$$
\mathrm{V}_{b \ell}=\frac{1}{2 \pi} \int_{-\infty}^{+\infty} d \omega^{\prime} Z_{\|}\left(\omega^{\prime}\right) \tilde{I}_{\ell}\left(\omega^{\prime}\right)
$$

where $Z_{\|}(\omega)$ is the longitudinal impedance, and $\tilde{I}_{l}(\omega)$ is the Fourier transform of the coherent current signal for mode $\ell$. The beam-induced voltage adds another effective driving term proportional to the coherent motion to the right hand side of Eq. (2). Jowett et. al. have found the self-consistent BTF for an inductive impedance to be[3]

$$
\frac{\left|\dot{\bar{\tau}}_{\ell}\right|}{g_{0}}=\frac{1}{\frac{1}{I}+K}
$$

where $K \propto j \bar{I}\left|\frac{z_{\|}}{n}\right|$ and $\bar{I}$ is the DC current. The synchrotron frequency in Eq. (5) must also be modified to include effects due to potential well distortion.

\section{Results}

\section{Relative Bunch Phase Measurements}

Since the individual bunch motions are recorded, the relative phase of the bunches can be examined. Shown in figure 1 is the measured phase difference between bunches while the beam was being excited at the mode frequency given by Eq. (1). The phase difference has been corrected for the time delay between bunch passage at the detector.
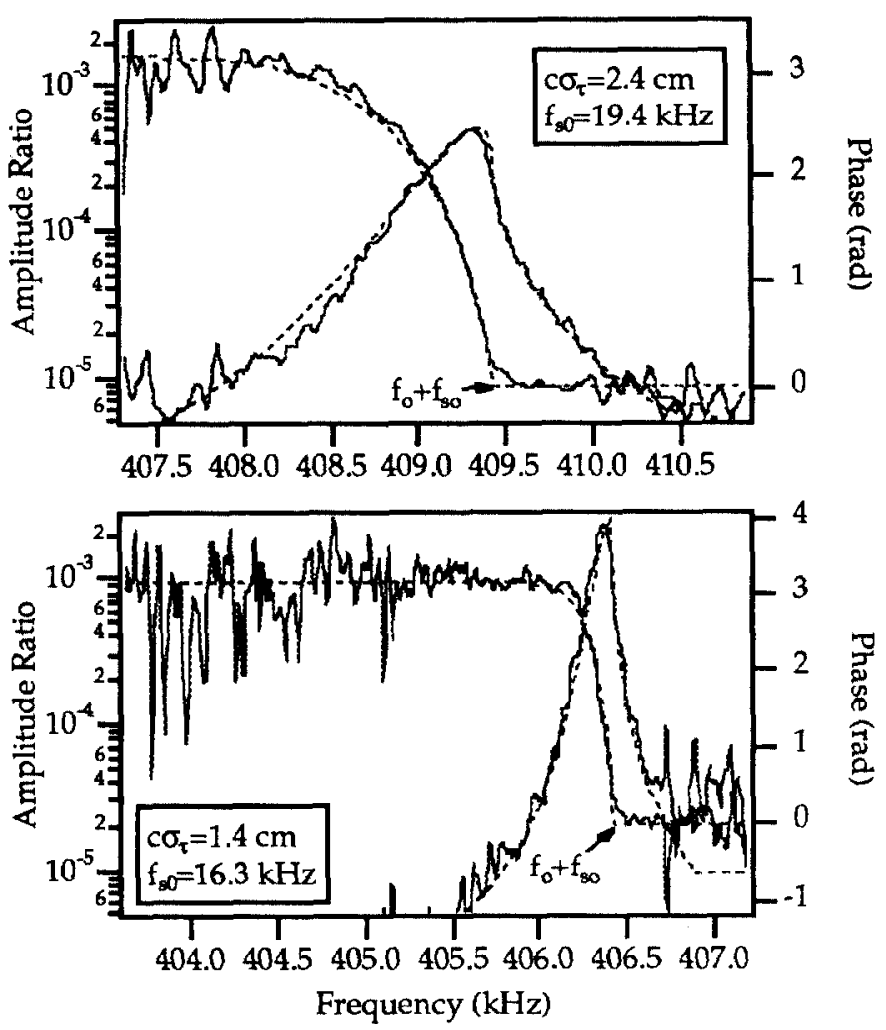

Figure 2: Low current transfer function measurements at 2 bunch lengths. Dashed curves are calculated from Eq. (4)

\section{Low Current Measurements}

In order to verify the model of the coherent response we made BTF measurements at several bunch lengths at low currents where the effect of the impedance can be ignored. Shown in figure 2 are typical BTF measurements for mode $\ell=1$ measured with a bunch lengths of $c \sigma_{\tau}=2.4 \mathrm{~cm}$ and $1.4 \mathrm{~cm}$ and bunch currents of $\sim 0.35 \mathrm{~mA} /$ bunch for 3 bunches. Dashed curves show the phase and amplitude response calculated from $\mathrm{Eq}$. (4). Measurements of the FM sidebands in the RF cavity without beam were used to determine the amplitude of the driving term, $g_{0}$. Transfer function measurements of the RF cavity itself showed the various cavity feedback loops had no effect on the BTF other than a slow dependence of the amplitude of the effective phase modulation on frequency. Care was taken to minimize the phase modulation in order not to distort the equilibrium bunch distribution.

\section{High Current Measurements}

The dominant narrowband longitudinal impedance in CESR is the fundamental mode of the RF cavity. Since the revolution frequency is large compared to the bandwidth of the fundamental mode, only the $\ell=0$ multibunch mode will interact with the cavity. This is commonly known as the Robinson effect. It is interesting to compare the BTF 
of the $\ell=0$ mode with a multibunch mode not interacting with the cavity fundamental mode but potentially interacting with other impedances such as parasitic higher modes of the cavity. Figure 3 shows the $\mathrm{BTH}^{\mathrm{T}}$ at three current levels for the $\ell=0$ and figure 4 shows the corresponding measurements for the $\ell=1$ mode for 3 bunches. The $\ell=0$ mode is strongly affected by the cavity and shows significant broadening of its transfer function due to interaction with the cavity impedance. The $\ell=1$ mode shows a small colerent tune shift and some distortion of the phase response. The behavior of all multibunch modes for 3 and 7 bunches, excluding $\ell=0$, is practically identical. Complete analysis of the measurements is in progress.

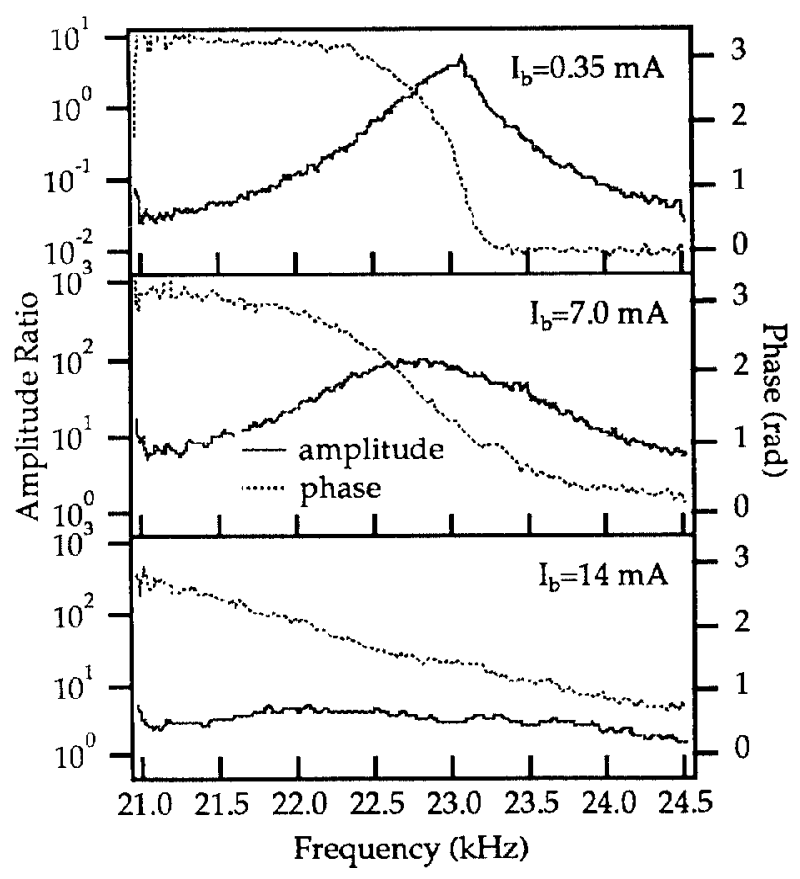

Figure 3: Beam transfer function vs. current for mode $\ell=0$.

\section{Conclusions}

Low current BTF measurements show good agreement with analytic calculation of the cohcrent response. High current measurements on various multibunch modes indicate very little impedance interaction except through the RF cavity fundamental mode. It may be possible to study single bunch effects such as potential well distortion using the BTF on multibunch modes which are not affected by the fundamental mode. Because the current dependence shown in fig. 4 is small, further analysis is necessary to obtain the effect of the impedance quantitatively. I would like to thank Richard Talman and Raphael Littauer for many thoughtful discussions.

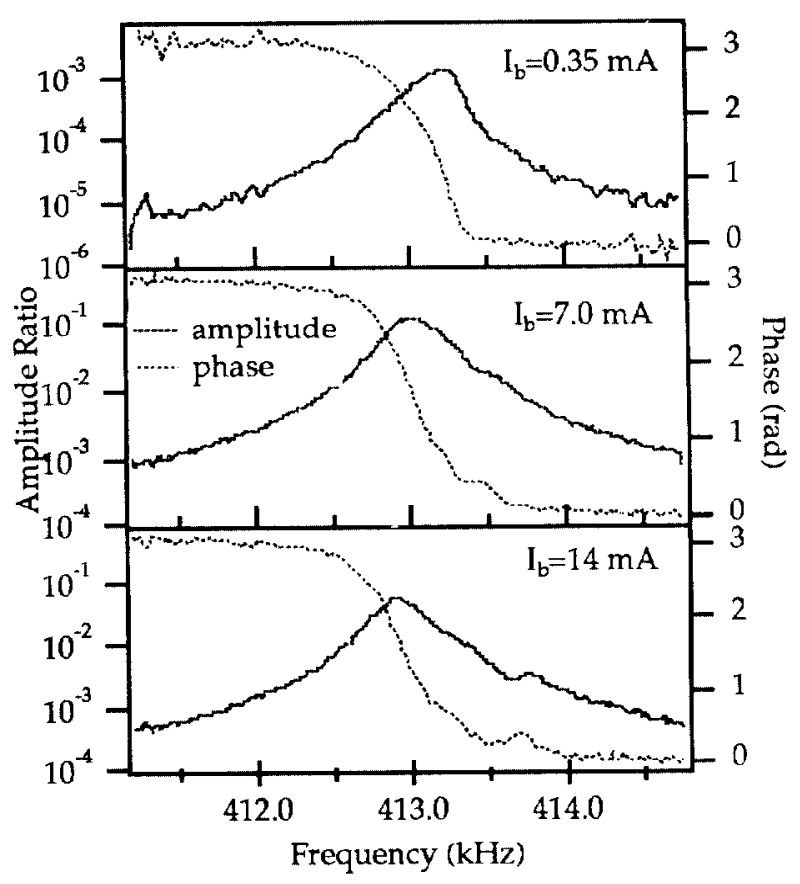

Figure 4: Beam transfer function vs. current for mode $\ell=1$.

\section{References}

[1] A. Hofmann and B. Zotter, IEEE Trans. Nucl. Sci. NS 24-3 pi487 (1977).

[2] J. Borer et. al., IEEE Trans. Nucl. Sci. NS-26, No. 3, (1977).

[3] J. M. Jowett, A. Hofmann, K. L. F. Banc, P. L. Morton, W. Spence, and R. Stege, in Proc. of European Particle Accelerator Conf., Nice, France (1990).

[4] S. Peggs, C. Saltmarsh, R. Talman, SSC-169 (1987).

[5] J. LeClare, CERN 87-03 (1987), LNS-043 and LNS-044 (1980).

[6] H. Hereward, in Theoretical Aspects of the Behavior of Beams in Accelerators and Storage Rings, Erice, (1977). 